\title{
Criminologie
}

\section{De la comparution à la décision pénale, le profil judiciaire de plus de 1500 femmes}

\section{Andrée B. Fagnan}

Volume 25, numéro 1, 1992

Les femmes et le contrôle pénal, questions féministes

URI : https://id.erudit.org/iderudit/017316ar

DOI : https://doi.org/10.7202/017316ar

Aller au sommaire du numéro

\section{Éditeur(s)}

Les Presses de l'Université de Montréal

ISSN

0316-0041 (imprimé)

1492-1367 (numérique)

Découvrir la revue

Citer cet article

Fagnan, A. B. (1992). De la comparution à la décision pénale, le profil judiciaire de plus de 1500 femmes. Criminologie, 25(1), 87-100.

https://doi.org/10.7202/017316ar
Résumé de l'article

The Elizabeth Fry Society of Montreal conducted a study aimed at describing the judicial profile of women defendants from their appearance to their sentencing. This article presents the principal results of this study which concerns more than 1500 women who appeared, in 1987, before the Quebec Criminal Court, at the Court houses at Montreal and Longueuil. Whether more than half these women are under 30 years of age, that they have never been charged before, that they are charged on one court only and of crimes against property, that they plead guilty and receive a probation order, very often the course of women through the judicial process is far from being linear. Once caught up in the system of judicial surveillance, there seems to be a sort of vicious circle established, where the system feeds the system, where the demeaning of the system becomes the offence that keeps these women within the system. 
DE LA COMPARUTION À LA DÉCISION PÉNALE, LE PROFIL JUDICIAIRE DE PLUS DE 1 500 FEMMES Andrée B. Fagnan ${ }^{1}$

The Elizabeth Fry Society of Montreal conducted a study aimed at describing the judicial profile of women defendants from their appearance to their sentencing. This article presents the principal results of this study which concerns more than 1500 women who appeared, in 1987, before the Quebec Criminal Court, at the Court houses at Montreal and Longueuil. Whether more than half these women are under 30 years of age, that they have never been charged before, that they are charged on one court only and of crimes against property, that they plead guilty and receive a probation order, very often the course of women through the judicial process is far from being linear. Once caught up in the system of judicial surveillance, there seems to be a sort of vicious circle established, where the system feeds the system, where the demeaning of the system becomes the offence that keeps these women within the system.

\section{INTRODUCTION}

Soucieuse d'approfondir sa compréhension des phénomènes de judiciarisation afin de poursuivre au mieux son objectif d'aide aux femmes confrontées à la justice pénale, la Société Elizabeth Fry de Montréal a mené une étude qui vise à décrire le profil judiciaire des femmes accusées, de la comparution à la décision pénale.

Après un bref rappel du contexte dans lequel elle se situe et de la méthodologie utilisée pour sa réalisation, nous rapportons les principaux résultats de cette étude, que nous avons faite pour la Société Elizabeth Fry de Montréal, sur le cheminement judiciaire de plus de 1500 femmes.

En conclusion, nous ferons état de quelques réflexions auxquelles ont donné lieu ces résultats tant en regard de la recherche que de la pratique.

1. Agente de recherche, Centre international de criminologie cornparte, Université de Montréal, case postale 6128, Succ. * A *, Montreal (Québec) H3C $3 J 7$ 


\section{TEXTES ET CONTEXTE}

Le rapport des femmes à la justice pénale constitue le contexte général de l'étude présentée ici. Un nombre croissant d'auteures se sont intéressées à cette question, au Canada, durant la dernière décennie. Rappelons la contribution de certaines d'entre elles.

Leurs recherches ont porté entre autres sur le portrait socio-économique des femmes justiciables (Boyle et al., 1985; Johnson, 1987; MacKinnon et Johnson, 1986) et sur les manifestations de la criminalité des femmes (Bertrand, 1979, 1983; Cousineau et al., 1986; Laberge, 1985-1986; MacKinnon et Johnson, 1986; Savard et Langelier-Biron, 1986). Il semble difficile, notamment à cause d'un manque de données statistiques pertinentes, d'établir un portrait exhaustif des femmes judiciarisées, mais dans l'ensemble des études, elles apparaissent comme plutôt jeunes et issues des classes sociales les moins favorisées. Par ailleurs, un état de fait est maintenant bien connu: peu de femmes commettent peu de crimes et encore moins de crimes graves. Devant ces constatations, la question de la détermination de la peine, point névralgique du processus pénal, devient, dans le cas des femmes, tout à fait incontournable. Des auteures se sont aussi penchées sur cette grave question. Elles traitent particulièrement de la discrimination dont les femmes font l'objet à différents moments du processus pénal (quand ce n'est pas le Code criminel in se qui en est jugé une source) et de la surpenalisation que cela entraînerait pour elles la plupart du temps (Bertrand, 1983; Boyle et al., 1985; Collette-Carrière et LangelierBiron, 1983; Hamelin, 1989; LaPrairie, 1984; Parent, 1986).

La complexité du système de justice pénale se pose aussi comme un élément contextuel important de la présente étude. Du délit à la peine, l'appareil de justice déploie toute une kyrielle de mécanismes dont les rouages tiennent à la fois des lois écrites, des règles administratives, des pratiques judiciaires et des differentes personnes (justiciables, victimes, témoins, policiers, avocats, juge, etc.) qui y interviennent (Pires et Landreville, 1985; Poirier, 1984). Il n'est pas simple de comprendre comment la justice opère quand tant de facteurs entrent en jeu, et les statistiques officielles, dont les modes d'enregistrement varient selon les différentes étapes du processus judiciaire, demeurent des outils limités pour le faire (Adelberg, 1985; Bertrand, 1979; Bertrand et al., 1984; Chapman, 1980; Johnson, 1987; Pires, 1987).

Too Few to Count, titre justement choisi par Adelberg et Currie en 1987, est devenu, depuis, une expression utile pour qui s'intéresse à la 
question des femmes et à la question pénale. Dans ce contexte où des femmes se retrouvent à la fois retenues et occultées par l'appareil de contrôle pénal, l'étude rapportée ici est en quelque sorte une mission de reconnaissance.

\section{LA MÉTHODOLOGIE}

Les données de l'étude dont il est fait état ici recouvrent une partie importante du cheminement judiciaire: de la comparution au prononcé de la sentence, voire à l'imposition de la peine. L'un des avantages de l'étude réside dans le fait qu'elle analyse des données qui concernent le cheminement de mêmes justiciables en cours de processus pénal.

L'étude porte sur un échantillon bien précis: les femmes qui ont comparu à la Cour du Québec, chambre criminelle, des palais de justice de Montréal et de Longueuil, du $1^{\mathrm{er}}$ janvier au 31 décembre 1987.

Les dossiers des femmes ayant comparu pendant l'année choisie pour l'étude ont été repérés au plumitif de la Cour. Comme un certain nombre de femme cumulent plus d'un dossier dans cette même année, c'est le plus récent dossier qui a été examiné comme objet principal de l'étude. Les résultats qui suivent portent donc sur 1571 cas, c'est-à-dire 1571 femmes différentes, accusées chacune sous au moins un chef.

Les données cueillies à même le plumitif fournissent des informations relatives aux antécédents judiciaires, aux chefs d'accusation, à la présence ou non d'un avocat de la defense, à certaines procédures comme l'enquête sur cautionnement, l'ordonnance d'examen mental et l'ordonnance de rapport présentenciel, aux plaidoyers de culpabilité ou aux décisions rendues par la cour en regard des accusations et aux peines. En dehors des données précitées sur le processus judiciaire comme tel, et mis à part le sexe, la seule autre information qui a été retenue pour l'étude est la date de naissance de chacun des sujets.

Ces données ont été transcrites et codées sur une grille élaborée à cet effet, puis saisies par informatique et traitées de manière à donner lieu à des résultats statistiques de nature descriptive. Ainsi se dessine le profil des femmes justiciables au fil de leur cheminement dans le processus judiciaire. Certaines variables sont aussi analysées les unes en fonction des autres de manière à identifier, à l'intérieur de ce processus, des facteurs d'influence sur le parcours judiciaire des femmes. 


\section{LES RÉSULTATS}

\section{L'âge des femmes accusées}

Les femmes accusées, dans la population étudiée ici, sont plutôt jeunes, c'est-à-dire qu'une majorité $(54,3 \%)$ d'entre elles ont moins de 30 ans. La médiane se situe à 28 ans et la moyenne est de 31 ans.

Il y a légèrement plus de "moins de 30 ans " parmi celles qui sont accusées de plus d'un chef, mais sur les 19 femmes accusées de plus de 20 chefs, 13 sont âgées de 30 ans et plus. Pour trois types de délits, les écarts sont particulièrement marqués entre les deux groupes d'âge: à la catégorie "complot ", plus des trois quarts des accusées ont moins de 30 ans, et de même pour les délits reliés à la drogue, on retrouve surtout des jeunes $(68 \%)$; par ailleurs, pour ce qui est des délits reliés à la conduite automobile, 64 pour cent des femmes qui en sont accusées ont 30 ans ou plus. La tendance veut que ce soit surtout les plus jeunes qui comparaissent sous arrestation et qui se voient refuser un cautionnement mais que ce soit surtout les « 30 ans et plus » qui se présentent en cour sans avocat et pour qui on ordonne un examen mental.

Un résultat peut surprendre: selon le sens commun et la logique du déroulement dans le temps, on pouvait s'attendre à ce que les résultats établissent un lien fort entre le fait d'avoir des dossiers antérieurs et celui de faire partie du groupe des aînées, mais l'analyse indique plutôt le contraire. Les plus jeunes sont plus nombreuses à avoir des dossiers antérieurs, et chez les moins jeunes, 60,8 pour cent n'ont pas de dossiers antérieurs. Un biais méthodologique peut expliquer ce résultat en partie: les données sont issues du plumitif informatisé de la cour, qui date d'une quinzaine d'années à peine; ce plumitif résume donc toute la vie adulte des "moins de 30 ans ", tandis qu'il n'en couvre qu'une partie chez les plus âgées.

\section{Les accusations}

S'il arrive qu'une femme accusée le soit pour plus d'un chef, voire jusqu'à 98 chefs lors d'une même affaire, plus de la moitié $(53,3 \%)$ des femmes ne le sont que pour un seul.

Comme on peut le voir au tableau 1, les femmes qui ont comparu en 1987 à la Cour du Québec, chambre criminelle, aux palais de justice de Montréal et de Longueuil, pour le dossier étudié, sont accusées principalement en vertu du Code criminel, et ce sont de délits contre les biens qu'elles sont accusées en plus grand nombre, 43,8 pour cent 
(dont $18,5 \%$ de fraude). Les données de l'étude ne permettent pas, cependant, de déterminer le degré de gravité des délits contre les biens. Par exemple, dans les dossiers où il est question de vol, il n'est pas précisé s'il s'agit de vol de moins ou de plus de 1000 dollars.

On peut également remarquer que 13,8 pour cent des femmes étaient accusées en vertu des lois sur les aliments et drogues et sur les stupéfiants et que 1,0 pour cent l'étaient en vertu d'autres lois tel le Code de la sécurité routière (10), la Loi sur l'assurance-chômage (2), sur le ministère du Revenu (1), sur la Société des alcools (1) et même celle des cités et villes (1).

Tableau 1

Le nombre et le pourcentage de femmes accusées, selon les types d'infractions

\begin{tabular}{lcc}
\hline \multicolumn{1}{c}{ Types d'infractions } & Nombre de femmes & Pourcentage $^{*}$ \\
\hline Contre les biens & 688 & 43,8 \\
Contre les personnes & 261 & 16,6 \\
Autres infractions au Code crimine ${ }^{* *}$ & 229 & 14,6 \\
Aliments, drogues et stupefiants & 2171 & 13,8 \\
Complot & 184 & 11,7 \\
Mours & 132 & 8,4 \\
Conduite automobile & 88 & 5,6 \\
Autres lois & 15 & 1,0 \\
\hline
\end{tabular}

* Ce pourcentage est calculé sur le nombre total de femmes (1 571). Notons qu'une même femme peut être accusée sous plus d'un type d'infractions.

** Infractions relatives aux agents de la paix, entrave à la justice, méfait public, évasion, omission de comparaître, être en liberté sans excuse, crime d'incendie, défaut de se conformer à une ordonnance.

\section{Les procédures}

Les femmes dont nous avons examiné le parcours judiciaire cheminent à travers un processus plus ou moins long. Ainsi, 19,8 pour cent de celles à qui l'on impose une peine voient leur affaire se régler en moins d'un mois; pour la majorité de ce groupe, les choses se passeraient ainsi: les femmes comparaissent, plaident coupables, reçoivent leur sentence, et ce dans la même journée. Par ailleurs, le délai entre la comparution et l'imposition de la peine sera de un à trois mois pour 43,8 pour cent des femmes sentenciées, de quatre à six mois pour 23,4 pour cent d'entre elles et 13 pour cent verront ce processus durer sept mois et plus.

Tout au long de la durée de leur affaire en cour, plus de 90 pour cent des femmes utilisent les services d'un avocat. C'est surtout lors- 
qu'elles sont accusées en vertu d' " autres lois » ou d'infractions reliées aux mœurs ou à la conduite automobile que les femmes se présentent en cour sans avocat.

Alors que 61,8 pour cent des femmes accusées comparaissent en liberté, 36,5 pour cent d'entre elles sont sous arrestation au moment de leur comparution, et ce surtout pour des délits reliés à la drogue, aux «autres infractions au C.c. » et aux mœurs où elles sont particulièrement surreprésentées.

Lorsqu'une enquête sur cautionnement est demandée, dans 85,6 pour cent des cas, le cautionnement est accordé. Les femmes à qui on refuse un cautionnement sont particulièrement accusées $d$ ' autres infractions au C.c. » $(41,0 \%)$. Plusieurs d'entre elles, la grande majorité dans le cas présent (19 sur 25), sont accusées sous l'article 133 , c'est-à-dire pour évasion, omission de comparaître ou liberté sans excuse, et trois autres sont accusées sous l'article 666, soit le défaut de se conformer à une ordonnance.

Un autre élément de procédure qui a retenu notre attention, c'est l'ordonnance d'examen mental qui a été émise dans le cas de 24 (1,5\%) femmes. Le profil judiciaire de ces femmes diffère de celui de l'ensemble: elles sont plus âgées, comparaissent presque toujours sous arrestation (22 sur 24), se voient refuser plus souvent un cautionnement, sont surtout accusées de voies de fait et d' «autres infractions au C.c.», comptent trois des quatre femmes «remises au bon plaisir du lieutenant-gouverneur " (c'est-à-dire jugées malades mentales) et elles font quatre fois plus souvent l'objet d'un rapport présentenciel.

Cette dernière ordonnance, le rapport présentenciel, est, elle aussi, mesure d'exception: elle touche $83(5,3 \%)$ des cas étudiés. Ce groupe présente également des particularités par rapport à l'ensemble de l'échantillon: les femmes sont près de deux fois plus souvent accusées de délits contre les personnes (utilisation illégale d'armes, vol qualifié, tentative de meurtre, homicide involontaire coupable, enlèvement), « reconnues » coupables, condamnées à l'emprisonnement et pour des peines plus longues, et reçoivent près de cinq fois plus souvent une ordonnance d'examen mental.

\section{Les décisions}

Chacun des chefs d'accusation dont est accusée chacune des femmes échantillonnées (1 571) est susceptible de faire l'objet de l'une ou l'autre décision, tel qu'illustré au tableau 2. 
La majorité $(62,2 \%)$ des femmes de l'étude plaident coupables à l'un ou l'autre de leurs chefs d'accusation et 44,3 pour cent plaident coupables à tous leurs chefs d'accusation. On peut voir au tableau $3^{2}$ que les femmes qui plaident coupables le font plus souvent pour des accusations relatives à la drogue que pour les autres types de délits. De même, on remarque que les femmes utilisent majoritairement le plaidoyer de culpabilité lorsqu'elles sont accusées pour des affaires de drogue $(71,0 \%)$, mais aussi lorsqu'elles sont accusées d' « autres infractions au C.c." $(66.5 \%)$, de complot $(61,1 \%)$, de délits relatifs aux mœurs $(54,0 \%)$ et de fraude $(52,5 \%)$.

Tableau 2

Les décisions en regard des chefs d'accusation

\begin{tabular}{lcc}
\hline \multicolumn{1}{c}{ Décision* } & Nombre de femmes & Pourcentage \\
\hline Plaide coupable & 977 & 62,2 \\
Chefs retirés & 260 & 16,5 \\
Libération de chefs & 195 & 12,4 \\
Acquitté & 140 & 8,9 \\
Sursis ou arrêt des procédures & 96 & 6,1 \\
Reconnue coupable & 87 & 5,5 \\
\hline
\end{tabular}

* La décision est prise par l'accusée dans le cas où elle plaide coupable et par la cour dans les autres cas.

Par ailleurs, le tableau 3 indique que, parmi les 3,9 pour cent de femmes qui sont reconnues coupables de tous leurs chefs, c'est dans la catégorie des délits contre les personnes qu'elles se retrouvent le plus $(26,4 \%)$, mais selon les différents types d'infractions, notons que les femmes ne sont majoritairement $(57,2 \%)$ reconnues coupables que lors d'accusations relatives aux « autres lois».

De plus, soulignons que, lorsqu'elles ne plaident pas coupables, les femmes sont acquittées dans la proportion de 64,9 pour cent contre 35,0 pour cent qui sont reconnues coupables.

2. Afin de poursuivre les analyses, nous avons créé neuf catégories d'infractions mutuellement exclusives. Il s'agit des huit types d'infractions déjà vues; le neuvième, c'est la « fraude » qui aété dégagée de l'ensemble des délits contre les biens. Les sujets de l'étưde qui se trouvent sous chaque catégorie n'ont à leur dossier que des accusations relevant de cette même catégorie. Ainsi, le nombre total de femmes passe de 1571 à 1351 . 
Tableau 3

Le nombre et le pourcentage de femmes selon qu'elles ont plaidé coupables ou ont été reconnues coupables sur tous leur chefs, par type d'infractions

\begin{tabular}{|c|c|c|c|c|c|c|c|c|}
\hline \multirow[b]{2}{*}{$\begin{array}{c}\text { Types } \\
\text { d'infractions }\end{array}$} & \multicolumn{2}{|c|}{$\begin{array}{c}\text { Plaide } \\
\text { coupable }\end{array}$} & \multicolumn{2}{|c|}{$\begin{array}{l}\text { Reconnue } \\
\text { coupable }\end{array}$} & \multicolumn{2}{|c|}{ Autre* } & \multicolumn{2}{|c|}{ Total } \\
\hline & $\begin{array}{l}\text { Nom- } \\
\text { bre }\end{array}$ & $\begin{array}{c}\text { Pour- } \\
\text { centage }\end{array}$ & $\begin{array}{c}\text { Non- } \\
\text { bre }\end{array}$ & $\begin{array}{c}\text { Pour- } \\
\text { centage }\end{array}$ & $\begin{array}{l}\text { Nom- } \\
\text { bre }\end{array}$ & $\begin{array}{c}\text { Pour- } \\
\text { centage }\end{array}$ & $\begin{array}{l}\text { Nom- } \\
\text { bre }\end{array}$ & $\begin{array}{l}\text { Pour- } \\
\text { centage }\end{array}$ \\
\hline$\overline{\text { Contre les biens }}$ & 120 & $\begin{array}{c}18,4 \\
(38,4)\end{array}$ & & $\begin{array}{l}13,2 \\
(2,2)\end{array}$ & 186 & $\begin{array}{r}8,8 \\
(59,4)\end{array}$ & 313 & $\overline{23,2}$ \\
\hline Fraude & 125 & $\begin{array}{c}19,2 \\
(52,5)\end{array}$ & 1 & $\begin{array}{l}18,9 \\
(4,2)\end{array}$ & 103 & $\begin{array}{c}15,9 \\
(43,3)\end{array}$ & 238 & 17,6 \\
\hline $\begin{array}{l}\text { Contre les } \\
\text { personnes }\end{array}$ & 68 & $\begin{array}{c}10,4 \\
(34,7)\end{array}$ & 1 & $\begin{array}{l}26,4 \\
(7,1)\end{array}$ & 114 & $\begin{array}{c}7,6 \\
(58,2)\end{array}$ & 196 & 14,5 \\
\hline Mœurs & 68 & $\begin{array}{c}10,4 \\
(54,0)\end{array}$ & & $\begin{array}{c}1,9 \\
(0,8)\end{array}$ & 57 & $\begin{array}{c}8,8 \\
(45,2)\end{array}$ & 126 & 9,3 \\
\hline Complot & 11 & $\begin{array}{c}1,7 \\
(61,1)\end{array}$ & & $\begin{array}{c}0,0 \\
(0,0)\end{array}$ & 7 & $\begin{array}{c}1,1 \\
(38,9)\end{array}$ & 18 & 1,3 \\
\hline $\begin{array}{l}\text { Conduite } \\
\text { automobile }\end{array}$ & 9 & $\begin{array}{c}1,4 \\
(10,5)\end{array}$ & & $\begin{array}{c}3,8 \\
(2,3)\end{array}$ & 75 & $\begin{array}{c}11,6 \\
(87,2)\end{array}$ & 86 & 6,4 \\
\hline $\begin{array}{l}\text { Autres infractions } \\
\text { au C.c. }\end{array}$ & 111 & $\begin{array}{c}17,0 \\
(66,5)\end{array}$ & & $\begin{array}{l}13,2 \\
(4,2)\end{array}$ & 49 & $\begin{array}{c}7,6 \\
(29,3)\end{array}$ & 167 & 2,4 \\
\hline Drogues & 137 & $\begin{array}{c}21,0 \\
(71,0)\end{array}$ & & $\begin{array}{r}7,5 \\
(2,1)\end{array}$ & 52 & $\begin{array}{c}8,1 \\
(26,9)\end{array}$ & 193 & 14,3 \\
\hline Autres lois & 3 & $\begin{array}{c}0,5 \\
(21,4)\end{array}$ & & $\begin{array}{l}8 \quad 15,1 \\
(57,2)\end{array}$ & 3 & $\begin{array}{c}0,5 \\
(21,4)\end{array}$ & 14 & 1,0 \\
\hline TOTAL & 652 & 48,3 & 5 & 3,9 & 646 & 47,8 & 1351 & 100,0 \\
\hline
\end{tabular}

* Cette catégorie « autre » comprend les cas où les décisions ont été différentes pour chacun des chefs et (ou) n'entraînaient pas de culpabilité.

\section{Les peines}

Dans la panoplie de peines prévues (tableau 4), une même personne peut se voir imposer entre une et quatre de ces mesures pénales. De fait, plus de la moitié $(57,9 \%)$ des femmes sentenciées reçoivent plus d'une peine, et ce, particulièrement lors de délits de fraude.

La probation est la peine la plus souvent donnée aux femmes de l'étude. En effet, plus de la moitié $(58,3 \%)$ des femmes sentenciées se retrouvent en probation, et ce, dans près de 90 pour cent des cas, pour au moins un an. Comme il se doit, la probation ne vient pas seule: 73,9 pour cent doivent faire face à une mesure en sus mais d'autres, à 
deux ou trois mesures en sus. La probation est surtout ordonnée lors de délits reliés à la fraude et à la drogue.

Près de la moitié $(45,8 \%)$ des femmes sentenciées sont condamnées a payer une amende variant de 25 à 7500 dollars, et cela surtout lors d'accusations reliées à la catégorie «contre les biens » $(25,2 \%)$ et à la catégorie «drogues " $(21,3 \%)$. Cependant, lorsqu'elles ont à payer de petits montants d'amende, moins de 100 dollars, c'est surtout pour des infractions contre les biens, tandis que, lorsqu'elles doivent payer une amende de 1000 dollars et plus, c'est surtout pour des délits relatifs aux drogues.

\section{Tableau 4}

Le profil des femmes trouvées coupables ou ayant enregistré un plaidoyer de culpabilité pour au moins un chef d'accusation selon les catégories de peines

\begin{tabular}{lcc}
\hline \multicolumn{1}{c}{ Peines } & Nombre de femmes & Pourcentage \\
\hline Ordonnance de probation & 597 & 58,3 \\
Amende & 469 & 45,8 \\
Sentence suspendue & 288 & 28,1 \\
Emprisonnement & 171 & 16,7 \\
Ordonnance de confiscation du permis & 58 & 5,7 \\
de conduire & 51 & 5,0 \\
Ordonnance de confiscation des biens & 51 & 5,0 \\
Autre montant & 29 & 2,8 \\
Liberation inconditionnelle & 17 & 1,7 \\
Ordonnance de restitution & 16 & 1,6 \\
Emprisonnement de fin de semaine & 13 & 1,3 \\
Ordonnance d'interdiction de port & 6 & 0,6 \\
d'armes & & \\
Autres peines (travaux communautaires) & 6 & \\
\hline
\end{tabular}

* Ce pourcentage est calculé sur le nombre total de femmes sentenciées (l 024$)$. Notons qu'une même femme peut recevoir des sentences de plusieurs catégories.

Et la prison? Cent soixante et onze femmes, c'est-à-dire 16,7 pour cent de celles à qui on impose au moins une peine, sont condamnées à la prison, et 16 femmes, 1,6 pour cent des sentenciées, à de la prison de * fin de semaine ». Les femmes à qui on impose une peine de prison sont particulièrement surreprésentées lorsqu'elles sont accusées d' "autres infractions au C.c.". Et comme on le voit au tableau 5, les sentences d'emprisonnement de courte durée ( 1 mois ou moins) sont aussi, pour plus de la moitié $(51 \%)$, imposées pour des délits de la catégorie « autres infractions au C.c.". Nous avons vérifié sous quels chefs sont accusées les 49 femmes qui se trouvent dans cette situation. Trois le 
sont sous l'article 118 (infractions relatives aux agents de la paix), quatre sous l'article 666 (défaut de se conformer à une ordonnance), cinq sous l'article 387 (méfaits) et 37 sous l'article 133 (évasion, omission de comparaître).

Tableau 5

Le temps d'emprisonnement selon les types d'infractions

\begin{tabular}{|c|c|c|c|c|c|c|c|c|}
\hline \multirow[t]{2}{*}{$\begin{array}{c}\text { Types } \\
\text { d'infractions }\end{array}$} & \multicolumn{2}{|c|}{$\begin{array}{l}1 \text { mois ou } \\
\text { moins }\end{array}$} & \multicolumn{2}{|c|}{2 à 13 mois } & \multicolumn{2}{|c|}{24 d 30 mois } & \multicolumn{2}{|c|}{72 mois et plus } \\
\hline & $\begin{array}{c}\text { Nom- } \\
\text { bre }\end{array}$ & $\begin{array}{l}\text { Pour- } \\
\text { centage }\end{array}$ & $\begin{array}{c}\text { Nom- } \\
\text { bre }\end{array}$ & $\begin{array}{l}\text { Pour- } \\
\text { centage }\end{array}$ & $\begin{array}{c}\text { Nom- } \\
\text { bre }\end{array}$ & $\begin{array}{l}\text { Pour- } \\
\text { centage }\end{array}$ & $\begin{array}{c}\text { Nom- } \\
\text { bre }\end{array}$ & $\begin{array}{l}\text { Pour- } \\
\text { centage }\end{array}$ \\
\hline Contre les biens & 25 & $\begin{array}{r}26,0 \\
(65,8)\end{array}$ & 13 & $\begin{array}{r}32,5 \\
(34,2)\end{array}$ & 0 & $\begin{array}{r}0,0 \\
(0,0)\end{array}$ & 0 & $\begin{array}{r}0,0 \\
(0,0)\end{array}$ \\
\hline Fraude & 8 & $\begin{array}{r}8,3 \\
(36,4)\end{array}$ & 12 & $\begin{array}{r}30,0 \\
(54,5)\end{array}$ & 2 & $\begin{array}{r}33,3 \\
(9,1)\end{array}$ & 0 & $\begin{array}{r}0,0 \\
(0,0)\end{array}$ \\
\hline $\begin{array}{l}\text { Contre les } \\
\text { personnes }\end{array}$ & 5 & $\begin{array}{r}5,2 \\
(33,3)\end{array}$ & 6 & $\begin{array}{r}15,0 \\
(40,0)\end{array}$ & 2 & $\begin{array}{r}33,3 \\
(13,3)\end{array}$ & 2 & $\begin{array}{r}66,7 \\
(13,3)\end{array}$ \\
\hline Mœurs & 1 & $\begin{array}{r}1,0 \\
(50,0)\end{array}$ & 1 & $\begin{array}{r}2,5 \\
(50,0)\end{array}$ & 0 & $\begin{array}{r}0,0 \\
(0,0)\end{array}$ & 0 & $\begin{array}{r}0,0 \\
(0,0)\end{array}$ \\
\hline Complot & 1 & $\begin{array}{r}1,0 \\
(100,0)\end{array}$ & 0 & $\begin{array}{r}0,0 \\
(0,0)\end{array}$ & 0 & $\begin{array}{r}0,0 \\
(0,0)\end{array}$ & 0 & $\begin{array}{r}0,0 \\
(0,0)\end{array}$ \\
\hline $\begin{array}{l}\text { Conduite } \\
\text { automobile }\end{array}$ & 1 & $\begin{array}{r}1,0 \\
(100,0)\end{array}$ & 0 & $\begin{array}{r}0,0 \\
(0,0)\end{array}$ & 0 & $\begin{array}{r}0,0 \\
(0,0)\end{array}$ & 0 & $\begin{array}{r}0,0 \\
(0,0)\end{array}$ \\
\hline $\begin{array}{l}\text { Autres infractions } \\
\text { au C.c. }\end{array}$ & 49 & $\begin{array}{r}51,0 \\
(87,5)\end{array}$ & 6 & $\begin{array}{r}15,0 \\
(10,7)\end{array}$ & 1 & $\begin{array}{r}16,7 \\
(1,8)\end{array}$ & 0 & $\begin{array}{r}0,0 \\
(0,0)\end{array}$ \\
\hline Drogues & 6 & $\begin{array}{r}6,3 \\
(60,0)\end{array}$ & 2 & $\begin{array}{r}5,0 \\
(20,0)\end{array}$ & 1 & $\begin{array}{r}16,7 \\
(10,0)\end{array}$ & 1 & $\begin{array}{r}33,3 \\
(10,0)\end{array}$ \\
\hline TOTAL & 96 & 66,2 & 40 & 27,6 & 6 & 4,1 & 3 & 2,1 \\
\hline
\end{tabular}

\section{Les antécédents judiciaires}

Des 1571 femmes de l'échantillon, 42,3 pour cent ont des mises en accusation antérieures au dossier étudié et 81,4 pour cent d'entre elles se sont déjà vu imposer une sentence. La majorité $(65,4 \%)$ des femmes qui comparaissent en liberté n'ont pas d'antécédents judiciaires alors que la majorité $(54,4 \%)$ de celles qui comparaissent sous arrestation en ont. En outre, 73,1 pour cent des femmes qui se voient refuser un cautionnement ont des antécédents.

Parmi ces femmes qui ont des antécédents judiciaires, 43,3 pour cent n'ont qu'un seul dossier antérieur. Nous avons observé que plus les femmes cumulent de dossiers anterieurs, plus le nombre de chefs d'ac- 
cusation a tendance à être élevé dans leur dossier à l'étude. Il y a un lien entre le fait d'avoir des antécédents et celui d'être ici accusée relativement aux «autres lois", aux «autres infractions au C.c.» et aux mours. Quand elles sont acquittées sur tous leurs chefs dans le cas étudié, les femmes ont moins d'antécédents judiciaires $(33,0 \%)$ que l'ensemble $(42,3 \%)$, et a contrario, elles en ont plus $(52,6 \%)$ que la moyenne $(42,3 \%)$ lorsqu'elles sont reconnues coupables. Notons aussi que plus de femmes $(68,0 \%)$ que la moyenne générale $(42,3 \%)$ ont des antécédents lorsque tous leurs chefs d'accusation sont retirés lors du dossier à l'étude. La libération inconditionnelle est nettement moins accordée à celles qui ont des dossiers judiciaires antérieurs et, par ailleurs, ces dernières sont près de deux fois plus souvent condamnées à la prison $(61,4 \%)$ que celles qui n'en ont pas $(31,6 \%)$.

Quant à l'impact des peines antérieures sur celles imposées relativement au dossier étudié, notons que plus il y a de dossiers antérieurs comportant une amende, plus il est probable qu'une peine de prison soit imposée par la suite. La corrélation est encore plus forte entre une ordonnance de probation antérieure et une peine de prison ultérieure. Il y a aussi un lien très marqué entre le fait d'avoir eu une peine de prison antérieure et celui d'en avoir une au dossier étudié.

\section{CONCLUSION}

Cette étude éclaire notre compréhension du processus judiciaire par lequel passent les femmes. En effet, plutôt que de prendre comme unité d'analyse les infractions ou les sentences ou même les procédures de cour et de les traiter de manière isolée, les mêmes femmes ont été suivies tout au long de leur cheminement dans l'appareil judiciaire. C'est là la spécificité de l'étude.

Il se dégage de cette étude un profil judiciaire type: les femmes ont moins de trente ans, elle n'ont pas été condamnées antérieurement, elles sont accusées sous un seul chef et de délits contre les biens, elles utilisent les services d'un avocat pour leur défense, elles comparaissent en liberté, elles plaident coupables et elles reçoivent une ordonnance de probation. Cependant, en filigrane de ce portrait type, nous découvrons que les trajectoires des femmes dans le processus judiciaire sont loin d'être toujours aussi linéaires.

En effet, des femmes sont prises au piège de ce processus. Une situation particulièrement problématique ressort clairement de l'étude. 
L'analyse des données permet d'affirmer que les femmes qui, d'une part, sont accusées d'évasion, d'omission de comparaître, d'être en liberté sans excuse et de défaut de se conformer à une ordonnance et à qui, d'autre part, on impose une peine de prison d'un mois ou moins composent le quart des femmes à qui on impose une peine de prison. Ce sont aussi, dans près de la moitié des cas, des femmes accusées des mêmes infractions à qui on refuse un cautionnement. Voilà des réalités qui s'apparentent à ce qu'il est convenu d'appeler le phénomène des portes tournantes. Une fois dans l'engrenage de la surveillance judiciaire, il semble s'établir une sorte de cercle vicieux où le système nourrit le système, où déroger du système devient le délit qui maintient dans ses rouages. Ainsi, les femmes qui « sont » en prison sont-elles en bonne partie celles qui y « retournent »? Qu'il y ait un lien entre le fait de s'être déjà vu imposer une peine de prison et d'être condamnée à cette même peine lors du dossier étudié incite à répondre par l'affirmative. Du point de vue de la pratique, les résultats décrits ci-dessus encouragent un organisme comme la Société Élizabeth Fry à poursuivre le développement de ses programmes d'hébergement et d'aide à la sortie de prison. Mais ces résultats incitent également à questionner le bien-fondé de la peine de prison, particulièrement dans le cas des femmes.

D'autres résultats décrits dans l'étude suggèrent de poursuivre d'autres recherches, par exemple en reprenant une étude semblable sur la population de femmes qui comparaît à la Cour municipale pour mieux cerner la problématique de la « détermination de la peine » reliée à la criminalité «moins grave» qui est en grande part le fait des femmes; en menant des études de type «qualitatif» pour saisir les enjeux du plaidoyer de culpabilité; et, à partir d'un sous-échantillon de la présente étude, en vérifiant jusqu'à quel point les décisions finales apparaissant aux dossiers étudiés marquaient ou pas la fin d'un cheminement « judiciaire » (qu'advient-il des « sentences suspendues »? comment sont gérées les peines de probation et de prison et les défauts de payer une amende?).

Soulignons encore un autre point d'intérêt: le fait que seulement quatre femmes aient reçu une peine de travaux communautaires est un autre résultat qui peut sans doute alimenter des réflexions dans l'ordre de l'intervention communautaire. Les données ont été cueillies en 1987; en est-il toujours ainsi des mesures de rechange à l'emprisonnement? Est-ce faute de ressources? Les juges y sont-ils suffisamment sensibilisés? 
Entre le délit, commis ou pas, et la peine, " juste " ou pas, plusieurs facteurs de compréhension débordent le champ pénal. L'étude dont il vient d'être question ici, nous en sommes consciente, ne rend compte que des pratiques pénales et tait bien des réalités sur les rapports des femmes à la "norme » et sur les différents contrôles sociaux, pénaux, formels et informels qui régissent ces rapports. Afin de bien comprendre le parcours des femmes dans le système pénal, il importe de conduire aussi des études d'autres types qui resituent les questions pénales dans un contexte plus large.

\section{BIBLIOGRAPHIE}

ADELBERG, Ellen (1985), Les Femmes qui ont des démêlés avec la justice: une minorité oubliée, Ottawa, Association canadienne des Sociétés Élizabeth Fry, $27 \mathrm{p}$.

ADELBERG, Ellen et CURRIE, Claudia (1987), Too Few to Count: Canadian Women in Conflict with the Law, Vancouver, Press Gang Publishers, 253 p.

BERTRAND, Marie-Andree (1979), La Femme et le crime. Montréal, l'Aurore, 224 p.

BERTRAND, Marie-Andrée (1983), « Femme et justice: problème de l"intervention *, Criminologie, vol. XVI, $\mathrm{n}^{\circ} 2$, pp. 77-88.

BERTRAND, Marie-Andrée et al., (1984), Recension des écrits sur la pathologisation des comportements féminins et la victimisation des femmes, Montréal, Université de Montréal, École de criminologie, $123 \mathrm{p}$.

BOYLE, Christine L. M. et al. (1985), Un examen féministe du droit criminel, Ottawa, ministère des Approvisionnements el Services, $232 \mathrm{p}$.

CHAPMAN, Jane Roberts (1980), " The Treatment of Convicted Female Offenders in the Criminal Justice System $n$, in C.T. Griffiths (ed.), The Female Offender, Vancouver, Simon Fraser University, Criminology Research Centre, pp. 195-201.

COLLETTE-CARRIÈRE, Renée et LANGELIER-BIRON, Louise (1983), "Du côté des filles et des femmes, leur délinquance, leur criminalitê », Criminologie, vol. XVI, $n^{\circ} 2$, pp. $27-45$.

COUSINEAU, Marie-Marthe, LABERGE, Danielle et THÉORÊT, Bruno (1986), Prisons et prisonniers: une analyse de la détention provinciale québécoise durant la dernière décennie, Montréal, UQAM, 450 p.

HAMELIN, Monique (1989), Femmes et prison, Montréal, Méridien, 270 p.

JOHNSON, Holly (1987), a Getting Facts Straight: A Statistical Overvicw $n$, in Adelberg, Too Few to Count, Vancouver, Press Gang Publishers, pp. 23-46. 
LABERGE, Danielle, «L'absence signifiante: de l'évanescence des femmes en prison ", Resources for Feminist Research/Documentation sur la recherche féministe, vol. 13, $\mathrm{n}^{\circ} 4$ (décembre/janvier 1985/1986), pp. 4-6.

LAPRAIRIE, Carol (1984), « Selected Criminal Justice and Socio-Demographic Data

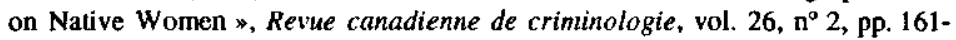
169.

MACKINNON, Judy et JOHNSON, Holly (1986), Women in Conflict with the Law: Facts and Figures, Ottawa, Ministère du Solliciteur génćral.

PARENT, Colette (1986), « La protection chevaleresque ou les représentations masculines du traitement des femmes dans la justice pénale $»$, Déviance et Société, vol. $10, \mathrm{n}^{\circ} 2$, pp. 147-175.

PIRES, Alvaro P. (1987), " La réforme pénale au Canada: l'apport đe la Commission canadienne sur la détermination de la peine », Criminologie, vol. $\mathrm{XX}, \mathrm{n}^{\circ} 2$, pp. 11-55.

PIRES, Alvaro P. et LANDREVILLE, Pierre (1985), « Les recherches sur les sentences et le culte de la loi ${ }^{2}$ L'Année sociologique, vol. 35, pp. 83-113.

POIRIER, Robert (1984), «La négociation des sentences du point de vue des avocats de la défense $*$, Criminologie, vol. $\mathrm{XX}, \mathrm{n}^{\circ} 2$, pp. 57-68.

SAVARD, Catherine et LANGELIER-BIRON, Louise (1986), Les Femmes auteurs de délits graves, Ottawa, Ministère du Solliciteur général, $153 \mathrm{p}$. 